\title{
Fiber-based multispeckle detection for time-resolved diffusing-wave spectroscopy: characterization and application to blood flow detection in deep tissue
}

\author{
G. Dietsche, M. Ninck, C. Ortolf, J. Li, F. Jaillon, and T. Gisler* \\ Fachbereich Physik, Universität Konstanz, 78457 Konstanz, Germany \\ ${ }^{*}$ Corresponding author: thomas.gisler@uni-konstanz.de
}

Received 18 April 2007; revised 30 August 2007; accepted 11 October 2007; posted 29 October 2007 (Doc. ID 82236); published 7 December 2007

\begin{abstract}
We present a technique for the measurement of temporal field autocorrelation functions of multiply scattered light with subsecond acquisition time. The setup is based on the parallel detection and autocorrelation of intensity fluctuations from statistically equivalent but independent speckles using a fiber bundle, an array of avalanche photodiodes, and a multichannel autocorrelator with variable integration times between 6.5 and $104 \mathrm{~ms}$. Averaging the autocorrelation functions from the different speckles reduces the integration time in diffusing-wave spectroscopy experiments drastically, thus allowing us to resolve nonstationary scatterer dynamics with single-trial measurements. We present applications of the technique to the measurement of arterial and venous blood flow in deep tissue. We find strong deviations both of the shape and characteristic decay time of autocorrelation functions recorded at different phases of the pulsation cycle from time-averaged autocorrelation functions.
\end{abstract}

OCIS codes: $\quad 030.5260,030.6140,170.6480$.

\section{Introduction}

Diffusing-wave spectroscopy [(DWS); also called diffuse correlation spectroscopy (DCS)] is the extension of quasi-elastic light scattering (QELS) to strongly multiply scattering media [1,2]. This method measures the autocorrelation function $g^{(2)}(\mathbf{r}, \tau)=$ $\langle I(\mathbf{r}, t) I(\mathbf{r}, t+\tau)\rangle /\langle I(\mathbf{r}, t)\rangle^{2}$ of the temporal fluctuations of the multiply scattered light intensity $I(\mathbf{r}, t)$ of the speckle pattern at the position $\mathbf{r}$. The temporal autocorrelation function of the scattered electric field $E(\mathbf{r}, t), g^{(1)}(\mathbf{r}, \tau)=\left\langle E^{*}(\mathbf{r}, t) E(\mathbf{r}, t+\tau)\right\rangle /\left\langle|E(\mathbf{r}, t)|^{2}\right\rangle$, is related to $g^{(2)}(\mathbf{r}, \tau)$ by the Siegert relation $g^{(2)}(\mathbf{r}, \tau)$ $=1+\beta\left|g^{(1)}(\mathbf{r}, \tau)\right|^{2}$ ( $\beta$ being the coherence factor) [3] and yields detailed information on the scatterer displacements within the sample, allowing e.g., discrimination of diffusive from subdiffusive motion $[4,5]$ or shear flow [6,7], or to detect dynamic heterogeneities even in the absence of static (transmission) contrast $[8,9]$. In contrast to QELS, the DWS field autocorrelation function depends on the optics of the medium by the reduced scattering coefficient $\mu_{\mathrm{s}}^{\prime}$ the absorption coefficient $\mu_{\mathrm{a}}$, and the boundary conditions for the photon transport.

Recently, DWS was introduced as a new method for diagnostics of biological tissue. In contrast to laser Doppler velocimetry or Doppler optical coherence tomography, DWS allows noninvasive probing of tissue at depths of several cm [10], owing to photoncounting detection and fast autocorrelators, enabling the measurement of autocorrelation functions decaying within no more than several microseconds.

Data from skin [11], tumors [12-15], skeletal muscle $[16,17]$, and the human brain cortex [18-20] suggest a strong coupling of the DWS signal to blood flow. While the driven erythrocyte motion, which is thought to be the origin of the DWS signal, is not Brownian in living tissue, experimental field autocorrelation functions are generally better described with diffusion than with (random) flow [19,21,22]. A recent 
comparison of DWS and arterial spin-label magnetic resonance data from the human calf muscle shows a correlation coefficient of approximately 0.7 between the effective particle diffusion coefficient extracted from DWS data and the blood flow velocity [17]. Noninvasive measurements on the human brain through the intact scalp and skull show that the effective diffusion coefficient in motor and visual cortices increases by $40 \%$ and by approximately $20 \%$, respectively, during stimulation [18-20], consistent with the typical functional increases of blood flow velocity measured by positron emission tomography.

Current DWS experiments from tissue require integration times of about 10-100 s, dictated by the low photon count rates (typically approximately $2 \mathrm{kHz}$ in a single speckle for $100 \mathrm{~mW}$ incident power) and short decay times (10-100 $\mu \mathrm{s}$ [19]) for source-receiver distances of $1.5-3 \mathrm{~cm}$. The transient non-Brownian motion of erythrocytes that is expected during the different phases of the pulsation cycle could thus be averaged out, resulting in apparently diffusive scatterer motion.

Resolving nonstationary scatterer dynamics below the pulsation time scale (typically $0.7-1 \mathrm{~s}$ in adult humans) requires detection methods for the DWS signal with a significantly improved signal-to-noise ratio (SNR). Using gated detection with a continuously integrating autocorrelator, pulsation-related nonstationary dynamics in deep tissue has been measured with a real-time resolution of approximately $200 \mathrm{~ms}$ [23]. However, this method requires averaging over many pulsation cycles and does not yield real-time data. Detection of a larger number of speckles with a single fiber with larger core diameter increases the average detected count rate, but reduces the coherence factor $\beta$, resulting in only slightly increased SNR of $g^{(1)}(\mathbf{r}, \tau)$ [24]. Using a CCD camera allows for parallel software autocorrelation of a large number of speckle spots and integration times not significantly larger than the decay time of $g^{(2)}(\mathbf{r}, \tau)$ $[25,26]$. While multispeckle DWS with a line-scan CCD camera (with a frame rate of $58 \mathrm{kHz}$ and 1024 pixels) allows probing of nonstationary dynamics with a real-time resolution of $10 \mathrm{~ms}$ [27], the shortest lag time of approximately $20 \mu \mathrm{s}$ presents a severe obstacle to monitoring dynamics in deep tissue.

In this paper we introduce a fiber-based multispeckle detection setup for measuring DWS autocorrelation functions with a real-time resolution of 6.5 $\mathrm{ms}$, using a bundle of optical fibers as mode-selective receivers, an avalanche photodiode (APD) array, and a multichannel, multitau correlator. Parallel autocorrelation of the intensity fluctuations of statistically equivalent, but independent, speckles, and averaging of the DWS signals from the different speckles enhances SNR for a given input laser power and predetection signal. Applications of the method to the real-time detection of nonstationary tissue dynamics within the fingertip, a large superficial vein, and the forehead in humans show characteristic strong pulsatile variations of the decay time of the DWS auto- correlation function. We find strong deviations both of the shape and the characteristic decay time of autocorrelation functions recorded at different phases of the pulsation cycle from autocorrelation functions measured by integration over many pulsation cycles.

\section{Methods}

\section{A. Multispeckle Setup}

We use an external-cavity diode laser operating at $\lambda=802 \mathrm{~nm}$ (TA100, Toptica) with a coherence length of approximately $100 \mathrm{~m}$ as a light source. To transport the laser light to the skin, a step-index multimode fiber (core diameter $50 \mu \mathrm{m}$, numerical aperture 0.22 ) is used. Its relatively short length of $2 \mathrm{~m}$ minimizes artifacts due to modal noise induced by subject-caused motions of the source fiber. In contrast to a single-mode fiber, the multimode fiber allows us to maximize the light flux delivered to the tissue without the need for additional shaping of the diode laser beam. To improve the SNR of the DWS signal while keeping the laser power at the skin below the safety limit of $4 \mathrm{~mW} / \mathrm{mm}^{2}$ [28], the illuminated area is increased to a diameter of approximately $4 \mathrm{~mm}$ by expanding the source beam through a glass plate placed between the skin and the source fiber end. Both the excitation fiber and the receiver fiber bundle (see below) are mounted rigidly on a rail that allows for continuous variation of the source-receiver distance. Source and receiver assembly are pressed gently onto the skin to minimize artifacts from relative motions between probe and tissue.

Our receivers consist of bundles with up to 32 single-mode fibers (SMF-28, Schäfter + Kirchhoff) with a cut-off wavelength of approximately $1260 \mathrm{~nm}$, numerical aperture 0.12 , and mode field diameter and cladding thickness of approximately 10 and $125 \mu \mathrm{m}$, respectively (see Fig. 1). Each fiber is $4 \mathrm{~m}$ long and is optically isolated with a black poly-vinyl chloride (PVC) cable. At the sample end, the isolation cable and the coating are removed over a length of 1 $\mathrm{cm}$, and the fiber ends are glued together with epoxy adhesive (Tra-Bond F112, Tra-Con) and inserted into a cylindrical poly-oxymethylene (POM) guide with $1 \mathrm{~mm}$ interior diameter [see Figs. 1(a) and 1(b)]. After curing, the front face of the receiver head is polished with a diamond paste with a granularity of $0.25 \mu \mathrm{m}$. For protecting the polished fibers from direct contact with the skin and for easier optical coupling through hair, we use a funnel-shaped collection tip with an integrated convex lens, which is screwed onto the fiber bundle [see Fig. 1(c)]. On the detector side each fiber is equipped with an FC-APC connector with an angular polish of $8^{\circ}$ to reduce the sensitivity of the transmission to bending and temperature variations. Because of variations of the fiber core diameters the number of transported modes in both polarizations varies from six to ten within the fiber bundle for the operating wavelength $\lambda=802 \mathrm{~nm}$. Fiber bending, e.g., from slow motions of the subject, introduces erratic jumps of the average photon count rate and erroneous normalization of the intensity autocorrela- 
(a)

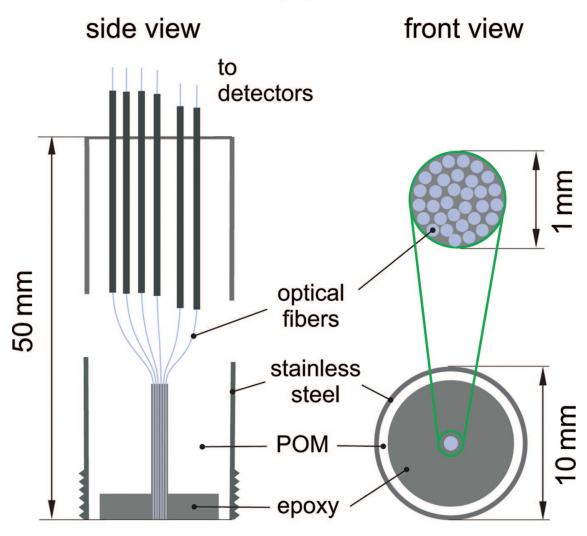

(b)

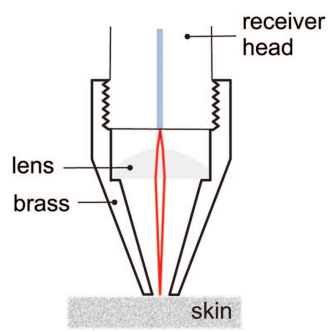

Fig. 1. (a) Side and top views of the multifiber receiver head, and (b) receiver head with attached collection optics. The receiver head consists of a stainless-steel sleeve holding a POM cylinder. Fewmode fibers, whose isolation jacket and coating were removed on a length of $1 \mathrm{~cm}$, are bundled inside a hole in the POM cylinder and fixed with epoxy adhesive. The collection tip with an integrated convex lens (b), screwed onto the receiver head (a), is used to image the speckle pattern from a small (diameter $<3 \mathrm{~mm}$ ) area on the skin onto the fiber faces. The red lines indicate the extremal rays from an $\mathrm{LP}_{01}$ fiber mode. The tip allows protecting the polished fiber faces from direct contact with the skin, which over time degrades the polish and the collection efficiency of the fibers. The conical brass funnel is used to move hair from the field of view.

tion function due to bending-dependent losses of the higher-order modes $\mathrm{LP}_{21}$ and $\mathrm{LP}_{02}$ [29]. These modes can be efficiently converted into nonpropagating leaky modes by wrapping the fibers over a cylinder $2.0 \mathrm{~cm}$ in diameter placed near the detectors, which results in bending-insensitive transport of six modes at $\lambda=802 \mathrm{~nm}$ for all fibers. Operating the fiber receiver with six modes reduces the coherence factor $\beta$ to $1 / 6 \approx 0.167$ (compared to $\beta=1$ for an ideal singlemode receiver) if the coupling efficiency is uniform across the modes. While the increased average predetection signal of the few-mode receiver enhances the SNR of the autocorrelation function by up to $40 \%$ [30] with respect to a single-mode fiber, the substantial benefit of the few-mode receiver comes from the increased SNR of the average photon count rate [24], which can be used, e.g., for assessing changes in tissue perfusion.

Photons are detected by four-channel APD modules (SPCM-AQ4C, Perkin-Elmer). Each APD channel is optically isolated from the others. The APD modules require a stable operating voltage (less than $2 \%$ peak- to-peak fluctuations for the $2 \mathrm{~V}$ supply), which is realized with two power supplies (PSAIG $5 \mathrm{~V} 40 \mathrm{~A}$, Mean Well). The output signal of each APD is a transistor-transistor logic (TTL) pulse $25 \mathrm{~ns}$ wide, which is sent to a 32 channel multitau correlator (correlator.com) designed for parallel computation of 32 photon-count autocorrelation functions. The integration times of the correlator can be chosen in multiples of $6.5 \mathrm{~ms}$, up to a maximum of $104 \mathrm{~ms}$. The 128 sampling times $\tau_{k}$ of the correlator are quasilogarithmically spaced, starting at $\tau_{1}=200 \mathrm{~ns}$. After a first block of 16 channels with a sampling time increment of $200 \mathrm{~ns}$, the latter is doubled every eight channels. The buffer of the correlator stores approximately 1000 normalized intensity autocorrelation functions $g_{i, j}^{(2)}(\tau)$, measured at subsequent times $t_{j}$, from each receiver channel $i \in\{1, \ldots, N\}$ before they have to be transferred to a personal computer (PC) (Pentium $3.2 \mathrm{GHz}$ ) via a universal serial bus (USB), where the bundle-averaged field autocorrelation function,

$$
\left\langle g_{j}^{(1)}(\tau)\right\rangle_{N}=\left[\frac{1}{N_{i}} \sum_{i=1}^{N}\left(g_{i, j}^{(2)}(\tau)-1\right) / \beta_{i}\right]^{1 / 2},
$$

is computed. The coherence factors $\beta_{i}$ for each receiver channel are determined beforehand with the same source-receiver geometry on a reference sample with similar characteristic decay time $1 / \Gamma$ and average count rate by fitting the exponential $\beta_{i} e^{-2 \Gamma \tau}$ to the trial-averaged reduced intensity autocorrelation function, $1 / M \sum_{j=1}^{M}\left(g_{i, j}^{(2)}(\tau)-1\right)$, computed from a sufficiently large number $M$ of experiments. This procedure compensates for nonidealities of the detectors (dead-time and dark counts [31]) and of the fiber receivers (see Subsection 2.B). A LABVIEW script is used to control the correlator and average the correlation functions.

The scatterer dynamics in the tissue is quantified by the decay time,

$$
\tau_{\mathrm{d}}=\int_{\tau_{1}}^{\tau_{2}} \mathrm{~d} \tau^{\prime}\left|\left\langle g_{j}^{(1)}(\tau)\right\rangle_{N}\right|^{2},
$$

with integration times $\tau_{1}$ and $\tau_{2}$ chosen to cover the full decay of $\left\langle g_{j}^{(1)}(\tau)\right\rangle_{N}$ without overweighting the baseline. While this parametrization does not explicitly discriminate between contributions from deep and from superficial layer dynamics to the field autocorrelation function from tissue, it is, unlike multilayer models [10,19], model-free since it does not require assumptions about tissue optical properties and scatterer dynamics.

\section{B. Characterization of the Setup}

\section{Coherence Factors and Collection Efficiency}

The sensitivity of the bundle-averaged field autocorrelation function to changes in the scatterer dynamics is not only limited by the photon counting 
statistics, but also by uncertainties in the coherence factors $\beta_{i}$. In real few-mode fiber receivers such uncertainties can arise from nonuniform coupling of the higher-order modes into the fiber, or due to different losses within the fiber for the fundamental and for the higher-order modes, e.g., due to tight bending. Nonuniform coupling can arise from a limited solid angle of observation when collection optics is used [24].

We have thus measured intensity autocorrelation functions $g_{i, j}^{(2)}(\tau)$ in transmission geometry from a suspension of polystyrene latex particles for each receiver channel of an $N=23$ fiber bundle. To reduce effects of incoherent background, we placed the sample, excitation fiber, and receiver head in a light-tight box. The average count rate was approximately $110 \mathrm{kHz}$, well below where detector dead-time effects have to be considered.

With the bare fiber receiver head, the value of the bundle-averaged coherence factor $\langle\beta\rangle_{N}=0.157$ is very close to the theoretical value $\beta=1 / 6 \approx 0.167$ for an ideal six-mode receiver with uniform transmission; the difference between experiment and theory arises from the detector dark counts of approximately $250 \mathrm{~Hz}$. The small standard deviation $\sigma_{\beta}=0.0025$ shows that the $L P_{01}$ and $L P_{11}$ modes are coupled into the fibers with nearly uniform efficiency. Bending the fibers in the bundle resulted in coherence factors with equal average $\left(\langle\beta\rangle_{N}=0.158\right)$, but slightly higher standard deviation $\left(\sigma_{\beta}=0.0071\right)$. With the collection tip attached to the receiver head, the average count rate is reduced by approximately $5.5 \%$ and the average intercept increases to a value $\langle\beta\rangle_{N}=0.163$. The standard deviation of $\beta_{i}$ is observed to increase to $\sigma_{\beta}$ $=0.0089$, indicating that the presence of the collection optics slightly reduces the coupling efficiency for the $\mathrm{LP}_{11}$ modes in some fibers. These results show that the receiver nonidealities are essentially due to the presence of the collection optics; the insensitivity of the coherence factors to fiber bending shows that the fiber wrapping effectively removes the higherorder modes $\mathrm{LP}_{21}$ and $\mathrm{LP}_{02}$. The insensitivity to fiber bending allows us to calibrate the receiver by measuring the coherence factors $\beta_{i}$ with sufficient accuracy on a stationary sample with known optical properties and dynamics. The correction for receiver nonidealities by Eq. (1) is particularly important for detecting physiological DWS signals with very small functional changes of $\tau_{d}$, such as from the human visual cortex [20]; here, the collection tip is essential for optimal signal collection through hair.

\section{Noise}

To test the performance of the multispeckle setup at low photon count rates and short integration times, we measured bundle-averaged field autocorrelation functions $\left\langle g_{j}^{(1)}(\tau)\right\rangle_{N}$ from an aqueous suspension of polystyrene latex spheres with an integration time $T^{\prime}=26 \mathrm{~ms}$ per autocorrelation function, using a receiver with $N=28$ fibers. Measurements were performed in transmission geometry through a slab-like

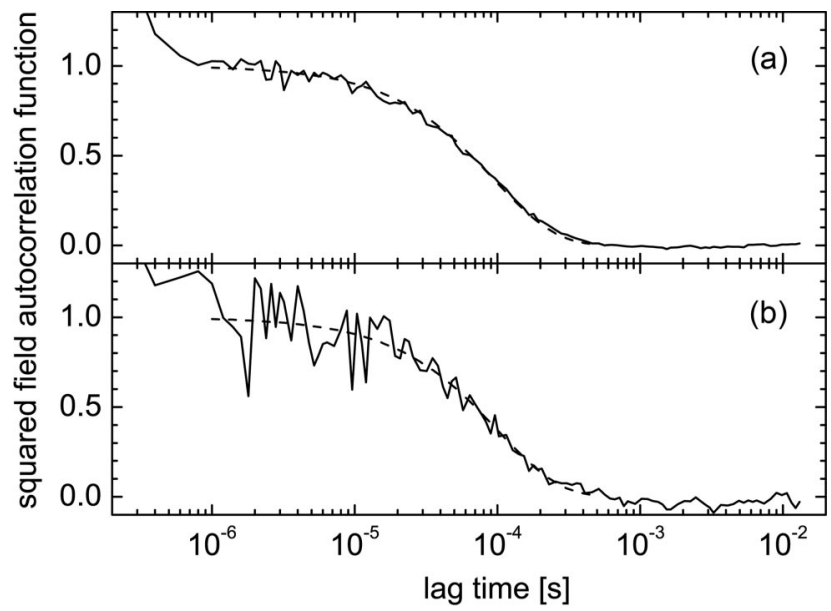

Fig. 2. Squared bundle-averaged field autocorrelation function $\left|\left\langle g_{j}^{(1)}(\tau)\right\rangle_{N}\right|^{2}$ from a suspension of polystyrene latex spheres with $N=28$ fibers and integration time $T^{\prime}=26 \mathrm{~ms}$, measured in transmission through a slab with thickness $1.0 \mathrm{~cm}$ and lateral dimensions $5.0 \times 5.0 \mathrm{~cm}^{2}$. Average count rate: $R=82 \mathrm{kHz}$. (a) Average over $M=20$ runs; (b) single run. Dashed curves: leastsquares fit of the prediction for the slab transmission geometry with expanded source and point-like receiver [32], with the reduced scattering coefficient $\mu_{\mathrm{s}}^{\prime}$ as a free parameter. The independently determined particle diffusion coefficient $D=9.2$ $\times 10^{-9} \mathrm{~cm}^{2} / \mathrm{s}$ and the absorption coefficient $\mu_{\mathrm{a}}=0.0223 \mathrm{~cm}^{-1}$ were used as fixed parameters. The values $\mu_{\mathrm{s}}^{\prime}=8.2 \mathrm{~cm}^{-1}$ (a) and $\mu_{\mathrm{s}}^{\prime}$ $=7.9 \mathrm{~cm}^{-1}$ (b) agree within $5 \%$ with Mie calculations [10].

cuvette with a thickness of $1.0 \mathrm{~cm}$ and lateral dimensions of $5.0 \times 5.0 \mathrm{~cm}^{2}$. We adjusted the photon count rate to an average value $R=82 \mathrm{kHz}$ per fiber, which is a value typical for experiments on the human head with a source-receiver distance of approximately 3 $\mathrm{cm}$. As shown in Fig. 2(a), the experimental data for $M=20$ runs (corresponding to a total integration time of $0.52 \mathrm{~s}$ ) agree very well with the theoretical prediction for the slab transmission geometry with expanded source and point-like receiver [32]. In single run data with integration time $T^{\prime}=26 \mathrm{~ms}$ [see Fig. 2(b)] the noise is still low enough that the value of $\mu_{s}^{\prime}$ obtained from a least-squares fit deviates from the prediction from Mie theory by no more than approximately $5 \%$.

For a field autocorrelation function decaying exponentially with a rate $\Gamma$, the noise model by Koppel $[22,33]$ predicts a standard deviation of the reduced intensity autocorrelation function, $g^{(2)}(\tau)-1$.

$$
\begin{aligned}
\sigma\left(\tau_{k}\right)= & \left(T_{k} / T^{\prime}\right)^{1 / 2} \\
& \times\left[\beta^{2} \frac{\left(1+e^{-2 \Gamma T_{k}}\right)\left(1+e^{-2 \Gamma \tau_{k}}\right)+2 k\left(1-e^{-2 \Gamma T_{k}}\right) e^{-2 \Gamma \tau_{k}}}{1-e^{-2 \Gamma T_{k}}}\right. \\
& \left.+\frac{2 \beta}{n_{k}}\left(1+e^{-2 \Gamma T_{k}}\right)+\frac{1}{n_{k}^{2}}\left(1+\beta e^{-\Gamma \tau_{k}}\right)\right]^{1 / 2}
\end{aligned}
$$

where $T_{k}=\tau_{k}-\tau_{k-1}$ is the width of the lag time bin $k$ and $n_{k}=R T_{k}$ is the average number of counts within the bin width $T_{i}$.

To make the correspondence with experiment, we estimate the standard deviation of the reduced 

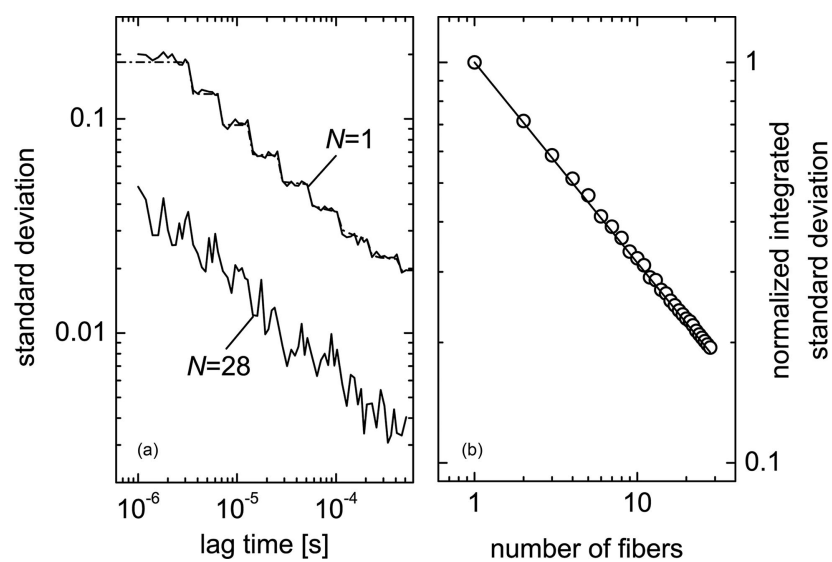

Fig. 3. (a) Standard deviation $\sigma(\tau ; N)$ of the reduced squared bundle-averaged field autocorrelation function $\langle\beta\rangle_{N}\left|\left\langle g_{j}^{(1)}(\tau)\right\rangle_{N}\right|^{2}$ for $N=28$ fibers and $N=1$ fiber, as a function of lag time $\tau$, measured on a polystyrene latex suspension. Sample and geometry are as in Fig. 2. The dashed-dotted curve is the prediction Eq. (3) for a single receiver fiber. The steps arise from the doubling of the sampling time increment [33,34]. (b) Normalized integrated standard deviation $s(N) / s(1)$ [see Eq. (5)] as a function of the number of fibers $N$ (symbols) and prediction $s(N) / s(1)=N^{-1 / 2}$ for statistically independent fibers (solid line).

bundle-averaged field autocorrelation function measured with $N$ fibers by

$$
\begin{aligned}
\sigma(\tau ; N)= & \langle\beta\rangle_{N}\left[\frac { 1 } { M - 1 } \sum _ { j = 1 } ^ { M } \left(\left|\left\langle\left\langle g^{(1)}(\tau)\right\rangle\right\rangle_{N, M}\right|^{2}\right.\right. \\
& \left.\left.-\left|\left\langle g_{j}^{(1)}(\tau)\right\rangle_{N}\right|^{2}\right)^{2}\right]^{1 / 2}
\end{aligned}
$$

As shown in Fig. 3(a), the experimental standard deviation for a single fiber $(N=1)$ closely follows the theoretical prediction Eq. (3). While the standard deviation of $g_{i, j}^{(2)}(\tau)-1$ depends on the coherence factor $\beta_{i}$, which slightly varies from one fiber to the other, the close agreement with theory reflects the low variance of the coherence factor.

Using a bundle with $N>1$ receiver fibers is expected to lead to a reduction in the standard deviation of $g^{(2)}(\tau)-1$ by a factor $N^{-1 / 2}$ with respect to a singlefiber receiver. Indeed, the measured standard deviation $\sigma(\tau ; N=28)$ for the bundle with the largest number of fibers available shows a functional dependence on $\tau$ very similar to the one for $N=1$ over the entire range of lag times, but with an amplitude reduced by a factor 0.183 , which is very close to the expected value $1 / \sqrt{28} \approx 0.189$ for independent fibers [see Fig. 3(a)].

To compare the dependence of the noise on the number of fibers used for averaging, we computed the integrated standard deviations,

$$
s(N)=\int_{\tau_{1}}^{\tau_{2}} \sigma(\tau ; N) \mathrm{d} \tau,
$$

with integration limits $\tau_{1}=1 \mu \mathrm{s}$ and $\tau_{2}=512 \mu \mathrm{s}$. As shown in Fig. 3(b), the standard deviations $s(N)$ scale as $N^{-1 / 2}$, which shows that the different detectors probe statistically equivalent but independent speckles. Consistent with the scaling of $s(N)$ is the negligible cross talk between fibers in the bundle, reflected by extremely low amplitudes of the cross-correlation function $\left(<10^{-5}\right)$ between the photon-count signals of neighboring fibers.

\section{Applications}

We illustrate the effectiveness of the multispeckle setup for following nonstationary tissue dynamics in real time by measurements of $\left\langle g_{j}^{(1)}(\tau)\right\rangle_{N}$ on the tip of the index finger, on the forearm, and over the left forehead of healthy volunteers recruited from faculty of the University of Konstanz. The study protocol was approved by the university's Ethical Review Board.

\section{A. Fingertip}

The fingertip is commonly used for optical pulse oxymetry using near-infrared transmission, as here the pulsatile variations of the concentrations of oxyand deoxy-hemoglobin in the arterial microvasculature are easily detected by measurements of the transmitted light intensity. The laser wavelength $\lambda$ $=802 \mathrm{~nm}$ is close to the isosbestic point of hemoglobin and deoxy-hemoglobin [35], which are the strongest absorbers in the types of tissue investigated here. Hence, variations of the average photon count rate reflect changes of the total hemoglobin concentration, $c_{\mathrm{tHb}}$, within the volume swept by the diffuse photon cloud. Source and receiver fibers were placed at a distance $\rho=1.4 \mathrm{~cm}$ parallel to the long axis of the index finger. In the strong absorption limit, the DWS signal thus probes the tissue dynamics at an average $\operatorname{depth}\left(\rho^{2} /\left(12 \mu_{\mathrm{a}} \mu_{\mathrm{s}}^{\prime}\right)\right)^{1 / 4} \approx 0.47 \mathrm{~cm}$ (assuming a reduced scattering coefficient $\mu_{\mathrm{s}}^{\prime}=19 \mathrm{~cm}^{-1}$ and absorption coefficient $\mu_{\mathrm{a}}=0.18 \mathrm{~cm}^{-1}$ [36]) [37]. Figure 4 shows

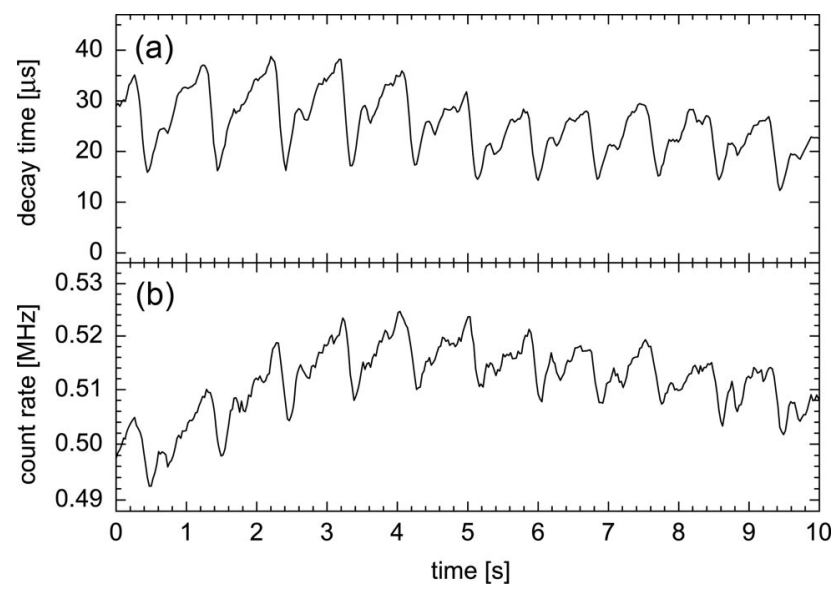

Fig. 4. (a) Decay time $\tau_{\mathrm{d}}$ and (b) average photon count rate $R$ measured on the tip of the index finger. Source-receiver distance: $1.4 \mathrm{~cm}$; integration time: $26 \mathrm{~ms}$. Number of receiver channels: $N$ $=23$. Data in (a) and (b) were smoothed by a $130 \mathrm{~ms}$ sliding average, corresponding to $14 \%$ of the average pulsation period. 


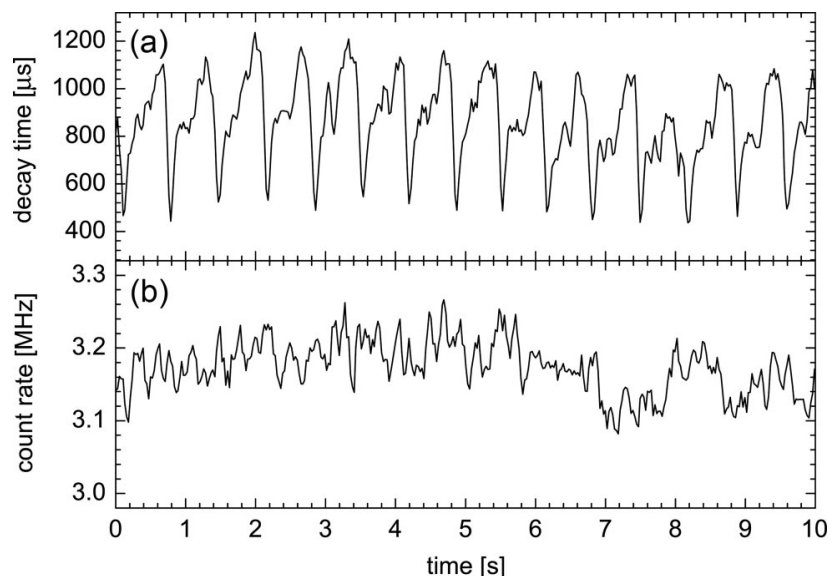

Fig. 5. (a) Decay time $\tau_{\mathrm{d}}$ and (b) average photon count rate $R$ measured over the medial cubital vein. Source-receiver distance: $1.6 \mathrm{~cm}$; integration time: $26 \mathrm{~ms}$. Number of receiver channels: $N$ $=8$. Data in (a) and (b) were smoothed by a $78 \mathrm{~ms}$ sliding average, corresponding to $11 \%$ of the average pulsation period. Experimental noise level in (b): $0.03 \%$ per data point.

the decay time $\tau_{d}$ and the average photon count rate $R$ measured with an acquisition time of $26 \mathrm{~ms}$ per correlation function, using $N=23$ receiver channels. Both $\tau_{\mathrm{d}}$ and $R$ show clear pulsatile variations; while the pulsatile variation of $R$ is between approximately $2 \%$ and $3.6 \%$ within one pulsation cycle, the one of $\tau_{d}$ is between approximately $190 \%$ and $240 \%$. Both the average count rate and the decay rate show additional fluctuations on a time scale of about $10 \mathrm{~s}$, which are due to vasomotion. The unsmoothed data show that the systolic minima of $\tau_{d}$ consistently precede the ones of $R$ (i.e., the maxima of $c_{\mathrm{tHb}}$ ) by $(49 \pm 14) \mathrm{ms}$.

\section{B. Venous Flow}

The DWS probe was placed with a source-receiver distance of $1.6 \mathrm{~cm}$ over the medial cubital vein at the elbow, along the direction of the vein. The arm was placed on a table at the height of the heart. The average decay time shows pulsatile variations between approximately $200 \%$ and $240 \%$ when the probe is placed directly over the vein [see Fig. 5(a)]. Displacement of the probe by approximately $1 \mathrm{~mm}$ off the vein strongly reduces the pulsatile variation of $\tau_{\mathrm{d}}$. Although the count rate variation of approximately $2.1 \%$ within the $10 \mathrm{~s}$ acquisition time window is similar to the one observed on the finger, periodic components indicative of volume pulsation cannot be discerned [see Fig. 5(b)].

\section{Forehead}

The DWS probe was placed with a source-receiver distance of $2.9 \mathrm{~cm}$ over the left forehead of a male subject sitting upright during the measurement. The average decay time $\tau_{\mathrm{d}}$ shows variations between $65 \%$ and $120 \%$ within a pulsation cycle [see Fig. $6(\mathrm{a})$ ]. The pulsatile variations of the average count rate $R$ are between $2 \%$ and $4 \%$ [see Fig. $6(\mathrm{~b})$ ]. The minima of $\tau_{\mathrm{d}}$ precede the ones of $R$ consistently by approximately

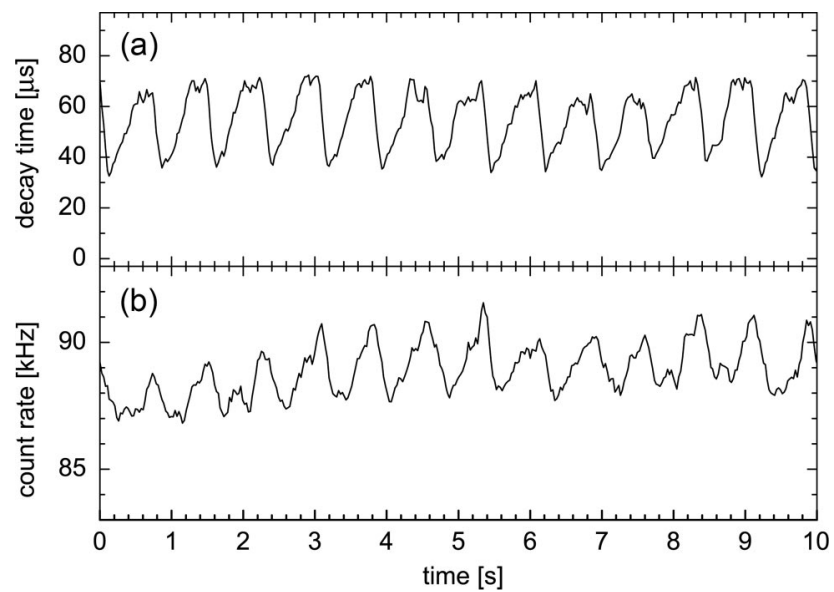

Fig. 6. (a) Decay time $\tau_{\mathrm{d}}$ and (b) average photon-count rate $R$ measured over the forehead. Source-receiver distance: $2.9 \mathrm{~cm}$; integration time: $26 \mathrm{~ms}$. Number of receiver channels: $N=14$. Data in (a) and (b) were smoothed by a $104 \mathrm{~ms}$ sliding average, corresponding to $14 \%$ of the average pulsation period of $758 \mathrm{~ms}$. Note the consistent phase lag of $175 \mathrm{~ms}$ between the minima of $\tau_{\mathrm{d}}$ and the ones of $R$. Experimental noise level in (b): $0.1 \%$ per data point.

$175 \mathrm{~ms}$. The pulsation waveform of $\tau_{\mathrm{d}}$ is rather symmetric, with a strong systolic minimum and a diastolic shoulder; the pronounced secondary minima in the diastolic phase, which are observed in the fingertip and vein, are not observed here.

\section{Discussion}

Our data show that the multispeckle DWS detection allows us to resolve pulsatile variations in the tissue dynamics in single recordings. The pulsatile waveform of $\tau_{d}$ measured on the fingertip shows a striking similarity to the one of the average count rate $R$, which reflects the variations in total hemoglobin concentration within the illuminated volume. The quantities $\tau_{\mathrm{d}}$ and $R$ show highly significant correlation: $r=0.34, p \ll 0.05$, for the raw data and $r=0.68$, $p \ll 0.05$, for the data smoothed with a $130 \mathrm{~ms}$ sliding average. During systole, the pulsation waveform of $\tau_{\mathrm{d}}$ shows a fast decrease to a primary minimum (reflecting fastest scatterer motion). The maximum of $\tau_{d}$ is reached after going through weaker secondary minima during diastole. These are reflected in the secondary minima in the average count rate $R$, which arise from reflections of the pressure wave due to the vascular impedance mismatch between arteries and capillaries [38]. We find that the pulsation rates extracted from the positions of the primary minima of $\tau_{\mathrm{d}}$ and $R$ are indistinguishable.

The variations of $R$ of between $2 \%$ and $3.6 \%$ are likely due to pulsatile variations of the arterial volume. An increase in $R$ then implies a decrease in both $c_{\mathrm{tHb}}$ and the erythrocyte concentration $c_{\mathrm{e}}$ within the illuminated volume. This results in opposing effects on the decay time of the DWS autocorrelation function: (i) the reduction in $c_{\mathrm{tHb}}$ leads to a decreased $\tau_{\mathrm{d}}$ due to a reduced absorption coefficient $\mu_{\mathrm{a}}$; (ii) assuming that the erythrocytes are the main mobile scatterers, a reduction in $c_{\mathrm{e}}$ tends to increase $\tau_{\mathrm{d}}$ due to the 
reduction in effective scattering events. Due to the linear relationship between $c_{\mathrm{tHb}}$ and $c_{\mathrm{e}}$ both effects are expected to result in variations in $\tau_{\mathrm{d}}$ that are small compared with the ones observed.

In addition, the consistent phase lag of approximately $49 \mathrm{~ms}$ between $R$ and $\tau_{\mathrm{d}}$ rules out a direct coupling of blood volume and DWS decay time by changes of absorption coefficient and erythrocyte concentration.

The data recorded over the medial cubital vein show that DWS resolves pulsation even when pulsatile variations of the average count rate are too small to be detected. The absence of pulsatile variations of $R$ reflects the low pressure in the venous system [37]. The pulsation waveform of $\tau_{\mathrm{d}}$ shows, similar to the fingertip data, a strong primary (systolic) minimum and weaker, but clearly resolved, secondary (diastolic) minima. In contrast to the fingertip waveform, the difference in $\tau_{\mathrm{d}}$ between primary systolic and secondary diastolic minima is stronger, leading to a more symmetric systolic dip. The absence of pulsatile variations in the average count rate indicates that the pulsatile variations of $\tau_{d}$ of between $200 \%$ and $240 \%$ are due to quasi-periodic variations of scatterer motion driven by the weak venous pulsation rather than due to changes in optical parameters.

The pulsatile variations of $\tau_{\mathrm{d}}$ measured over the left forehead of between $65 \%$ and $120 \%$ are considerably smaller than the ones measured on the index fingertip and over the medial cubital vein. Due to the relatively large source-receiver distance of $2.9 \mathrm{~cm}$, the DWS signal probes not only the skin, but also the skull and superficial parts of the brain. While skin is perfused by arterial vessels large enough to show pulsation, blood supply in the skull is mainly accomplished by capillaries in which blood flow is nonpulsatile. The skull perfusion is thus expected to lead to a slowly varying offset in $\tau_{d}$ and, hence, a weaker systolic-diastolic variation of $\tau_{\mathrm{d}}$. Both the phase lag of $23 \%$ of the pulsation period and its variance of approximately $52 \%$ are considerably higher than on the fingertip.

The strong pulsatile variations of the characteristic decay time $\tau_{\mathrm{d}}$, which is used to parametrize the decay of the field autocorrelation function, suggest that measuring DWS autocorrelation functions from tissue with integration times of many pulsation periods (required by the poor SNR of conventional singlefiber experiments) average over temporally heterogeneous, nonstationary dynamics. This averaging effect might explain why, despite the strong correlation of DWS signals with blood flow [17], the field autocorrelation function is described by diffusion rather than by (random) flow $[18,19,21]$.

To investigate whether the DWS autocorrelation functions differ in shape within a pulsation cycle, we compared $\left\langle g_{j}^{(1)}(\tau)\right\rangle_{N}$ at the systolic minima and at the diastolic maxima of $\tau_{\mathrm{d}}$, using the data measured on the index finger with an integration time of $26 \mathrm{~ms}$ (see Fig. 7). To reduce noise, we averaged the respective correlation functions from 11 pulsation cycles

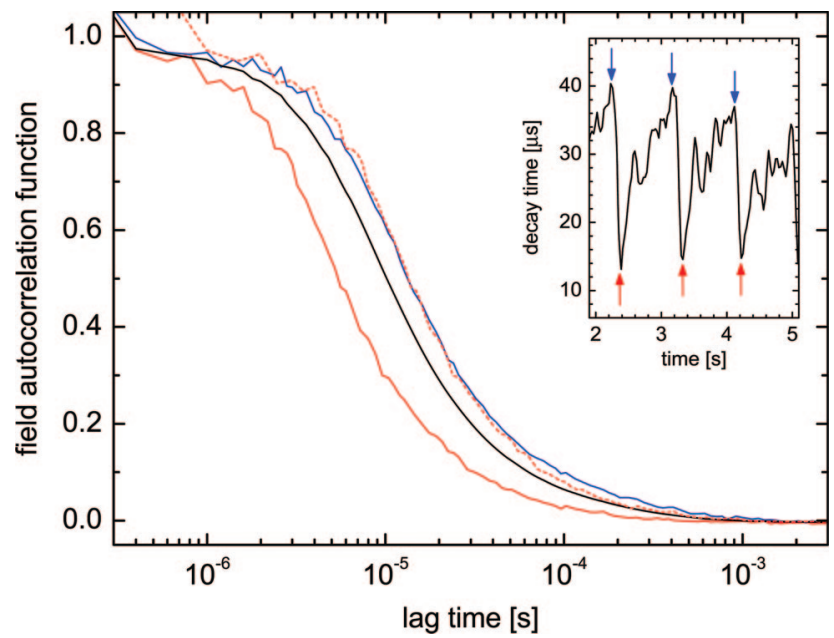

Fig. 7. Field autocorrelation functions $\left\langle g_{j}^{(1)}(\tau)\right\rangle_{N}$ as a function of lag time $\tau$ at the diastolic maxima (blue solid curve) and systolic minima (red curve) of $\tau_{\mathrm{d}}$ measured at the fingertip. Number of receiver channels: $N=23$. Integration time per field autocorrelation function: $26 \mathrm{~ms}$. Data are averages over 11 field autocorrelation functions measured at the maxima and minima of the $\tau_{\mathrm{d}}$ curve (blue and red arrows, respectively, in inset). Black curve: $\left\langle g_{j}^{(1)}(\tau)\right\rangle_{N}$ averaged over $10 \mathrm{~s}$. For easier comparison with the diastolic data, the systolic field autocorrelation function was shifted in time (red dashed curve). Note the larger curvature at short lag times of the field autocorrelation function recorded at the systolic minimum.

[38]. The positions of the systolic minimum and diastolic maximum, respectively, were determined by hand. As shown in Fig. 7, the field autocorrelation functions differ not only in their characteristic time scale but also in the shape of their decay. In particular, the field autocorrelation function at the systolic minimum is more strongly curved at short and at long lag times than the one at the diastolic maximum. With the source-receiver distance $\rho=1.4 \mathrm{~cm}$, the tissue at the index fingertip can be approximated as a semi-infinite homogeneous medium whose absorption coefficient $\mu_{\mathrm{a}} \approx 0.18 \mathrm{~cm}^{-1}$ is much smaller than the reduced scattering coefficient $\mu_{\mathrm{s}}^{\prime} \approx 19 \mathrm{~cm}^{-1}$. In this limit the field autocorrelation functions for different reduced scattering coefficients can be scaled onto each other, provided the scatterer dynamics is stationary [19]. The data measured at the fingertip for the systolic minimum and the diastolic maximum of $\tau_{\mathrm{d}}$ can, in contrast, not be scaled onto each other, which clearly shows that the pulsatile variation of the DWS autocorrelation function is not due to optical effects such as variations of $\mu_{\mathrm{s}}^{\prime}$, but due to nonstationary scatterer dynamics.

For the medial cubital vein and for the left forehead, we observe a similar lack of scaling between systolic and diastolic field autocorrelation functions. However, approximating the tissue at a superficial large vessel and at the head as a semi-infinite medium is not realistic; hence, the lack of scaling of the systolic and the diastolic correlation functions is more difficult to interpret than at the fingertip.

An essential prerequisite for the correct estimation of field autocorrelation functions by time-averaging 
correlators is the stationarity of the average photon count rate during the integration time. This condition is obviously not met when the count rate shows strong pulsatile variations, such as in the fingertip or on the forehead. In the fingertip, the maximal relative count rate drifts are observed on the systolic slopes, with typical values of approximately $0.5 \%$ within an integration time of $26 \mathrm{~ms}$. For the large count rates measured on the fingertip $(R \approx 0.5 \mathrm{MHz})$, the photon-counting noise within the integration time of $26 \mathrm{~ms}$ is of the order of $0.3 \%$, comparable to the drift. For the forehead data, the maximal systolic count rate drift is approximately $0.5 \%$ within $26 \mathrm{~ms}$, comparable to the situation at the fingertip. However, the photon-counting noise is much larger here (approximately $2.1 \%$ ), so that the count rate drift is only a minor contribution to the overall noise on the count rate. Under the experimental conditions encountered in human tissue (low average photon count rates, pulsation rates of approximately $1 \mathrm{~Hz}$ ), the presence of photon-count drift due to pulsation does not appear to seriously affect the quality of timeresolved DWS data, as shown by coherence factors from in vivo data that are practically indistinguishable from the ones from samples with stationary count rate and dynamics.

\section{Conclusions}

The present fiber-based multispeckle detection extends DSW to probing nonstationary fast scatterer dynamics with a real-time resolution of as small as $6.5 \mathrm{~ms}$. In comparison to camera-based multispeckle detection schemes that offer a similar real-time resolution, the use of APDs and a fast multichannel correlator offers superior resolution in lag time, thus allowing us to monitor samples with a vastly varying range of speckle fluctuation rates with a single setup. Using few-mode optical fibers particularly allows us to spatially separate the detection from the sample, which is, e.g., useful for tomographical DWS imaging [22]. Furthermore, fiber receivers allow us to collect scattered light from samples that are not accessed with ease, such as for endoscopical applications.

The main drawbacks of APDs, namely, their relatively large dead-time and the considerable afterpulsing, could be overcome by pseudo cross correlation; while this technique is prohibitive at present, complementary metal-oxide semiconductorbased photon-counting APD arrays with a greatly extended number of pixels and on-chip data processing could considerably increase both the sensitivity and the temporal resolution of multi-speckle DWS.

The time-resolved data from different types of biological tissue show pronounced pulsatile variations in the scatterer dynamics. However, the origin of the DWS signal in the different types of tissue, namely, the roles of erythrocyte flow velocity and shear deformations within the extravascular tissue, is at present not entirely clear. Here experiments on tissue phantoms with controlled optical and dynamical properties could lead to a more quantitative understanding of the DWS signal from real tissues. The temporal resolution and the high sensitivity to perfusion make the method especially attractive for monitoring perfusion of specific areas of the brain cortex following stimulation. In turn, the temporal resolution may be traded in for largely enhanced sensitivity in experiments with long integration times. This allows us to resolve very small functional changes of the DWS signal, as was recently demonstrated for the visual cortex [20].

We thank J. Zhu for help with the implementation of the correlator, M. Weiland for support with the receiver construction, E. Keller, A. Heinen, T. Binzoni, and M. Wolf for helpful discussions, and G. Maret for continuous support. This work is funded by the Center for Applied Photonics (CAP) Konstanz, the Deutsche Forschungsgemeinschaft (DFG), and the Landesstiftung Baden-Württemberg.

\section{References}

1. G. Maret and P. E. Wolf, "Multiple light scattering from disordered media: The effect of Brownian motion of scatterers," Z. Phys. B 65, 409-413 (1987).

2. D. J. Pine, D. A. Weitz, P. M. Chaikin, and E. Herbolzheimer, "Diffusing-wave spectroscopy," Phys. Rev. Lett. 60, 1134-1137 (1988).

3. B. J. Berne and R. Pecora, Dynamic Light Scattering (Krieger, 1990).

4. T. Gisler and D. A. Weitz, "Scaling of the microrheology of semidilute F-actin solutions," Phys. Rev. Lett. 82, 1606-1609 (1999).

5. B. S. Chae and E. M. Furst, "Probe surface chemistry dependence and local polymer network structure in F-actin microrheology," Langmuir 21, 3084-3089 (2005).

6. X.-L. Wu, D. J. Pine, P. M. Chaikin, J. P. Huang, and D. A. Weitz, "Diffusing-wave spectroscopy in a shear flow," J. Opt. Soc. Am. B 7, 15-20 (1990).

7. D. Bicout and G. Maret, "Multiple light scattering in TaylorCouette flow," Physica A 210, 87-112 (1994).

8. D. A. Boas, L. E. Campbell, and A. G. Yodh, "Scattering and imaging with diffusing temporal field correlations," Phys. Rev. Lett. 75, 1855-1858 (1995).

9. M. Heckmeier and G. Maret, "Visualization of flow in multiplescattering liquids," Europhys. Lett. 34, 257-262 (1996).

10. F. Jaillon, S. E. Skipetrov, J. Li, G. Dietsche, G. Maret, and T. Gisler, "Diffusing-wave spectroscopy from head-like tissue phantoms: influence of a non-scattering layer," Opt. Express 14, 10181-10194 (2006).

11. D. A. Boas, "Diffuse photon probes of structural and dynamical properties of turbid media: Theory and biomedical applications," Ph.D. dissertation (University of Pennsylvania, 1996).

12. C. Menon, G. M. Polin, I. Prabakaran, A. Hsi, C. Cheung, J. P. Culver, J. F. Pingpank, C. S. Sehgal, A. G. Yodh, D. G. Buerk, and D. L. Fraker, "An integrated approach to measuring tumor oxygen status using human melanoma xenografts as a model," Cancer Res. 63, 7232-7240 (2003).

13. U. Sunar, H. Quon, T. Durduran, J. Zhang, J. Du, C. Zhou, G. Q. Yu, R. Choe, A. Kilger, R. Lustig, L. Loevner, S. Nioka, B. Chance, and A. G. Yodh, "Noninvasive diffuse optical measurement of blood flow and blood oxygenation for monitoring radiation therapy in patients with head and neck tumors: a pilot study," J. Biomed. Opt. 11, 064021 (2006).

14. G. Yu, T. Durduran, C. Zhou, H.-W. Wang, M. E. Putt, H. M. Saunders, C. S. Sehgal, E. Glatstein, A. G. Yodh, and T. M. Busch, "Noninvasive monitoring of murine tumor blood flow during and after photodynamic therapy provides early assess- 
ment of therapeutic efficacy," Clin. Cancer Res. 11, 3543-3552 (2005).

15. G. Yu, T. Durduran, C. Zhou, T. C. Zhu, J. C. Finlay, T. M. Busch, S. B. Malkowicz, S. M. Hahn, and A. G. Yodh, "Realtime in situ monitoring of human prostate photodynamic therapy with diffuse light," Photochem. Photobiol. 82, 1279-1284 (2006).

16. G. Yu, T. Durduran, G. Lech, C. Zhou, B. Chance, E. R. Mohler, III, and A. G. Yodh, "Time-dependent blood flow and oxygenation in human skeletal muscles measured with noninvasive near-infrared diffuse optical spectroscopies," J. Biomed. Opt. 10, 024027 (2005).

17. G. Yu, T. F. Floyd, T. Durduran, C. Zhou, J. Wang, J. A. Detre, and A. G. Yodh, "Validation of diffuse correlation spectroscopy for muscle blood flow with concurrent arterial spin labeled perfusion MRI," Opt. Express 15, 1064-1075 (2007).

18. T. Durduran, G. Yu, M. G. Burnett, J. A. Detre, J. H. Greenberg, J. Wang, C. Zhou, and A. G. Yodh, "Diffuse optical measurement of blood flow, blood oxygenation, and metabolism in a human brain during sensorimotor cortex activation," Opt. Lett. 29, 1766-1768 (2004).

19. J. Li, G. Dietsche, D. Iftime, S. E. Skipetrov, G. Maret, T. Elbert, B. Rockstroh, and T. Gisler, "Non-invasive detection of functional brain activity with near-infrared diffusing-wave spectroscopy,” J. Biomed. Opt. 10, 044002 (2005).

20. F. Jaillon, J. Li, G. Dietsche, T. Elbert, and T. Gisler, "Activity of the human visual cortex measured non-invasively by diffusing-wave spectroscopy," Opt. Express 15, 6643-6650 (2007).

21. C. Cheung, J. P. Culver, K. Takahashi, J. H. Greenberg, and A. G. Yodh, "In vivo cerebrovascular measurement combining diffuse near-infrared absorption and correlation spectroscopies," Phys. Med. Biol. 46, 2053-2065 (2001).

22. C. Zhou, G. Yu, D. Furuya, J. H. Greenberg, A. G. Yodh, and T. Durduran, "Diffuse optical correlation tomography of cerebral blood flow during cortical spreading depression in rat brain," Opt. Express 14, 1125-1144 (2006).

23. J. Li, F. Jaillon, G. Dietsche, G. Maret, and T. Gisler, "Pulsation-resolved deep tissue dynamics measured with diffusing-wave spectroscopy," Opt. Express 14, 7841-7851 (2006).

24. T. Gisler, H. Rüger, S. U. Egelhaaf, J. Tschumi, P. Schurtenberger, and J. Rička, "Mode-selective dynamic light scattering: theory versus experimental realization," Appl. Opt. 34, 35463553 (1995).

25. A. P. Y. Wong and P. Wiltzius, "Dynamic light scattering with a CCD camera," Rev. Sci. Instrum. 64, 2547-2549 (1993).

26. L. Cipelletti and D. A. Weitz, "Ultralow-angle dynamic light scattering with a charge coupled device camera based multispeckle, multitau correlator," Rev. Sci. Instrum. 70, 32143221 (1999).

27. R. Bandyopadhyay, A. S. Gittings, S. S. Suh, P. K. Dixon, and D. J. Durian, "Speckle-visibility spectroscopy: A tool to study time-varying dynamics," Rev. Sci. Instrum. 76, 093110 (2005).

28. Deutsches Institut f. Normung e. V., Deutsche IndustrieNorm EN 60825-1: Sicherheit von Laser-Einrichtungen (Beuth, 2003).

29. E.-G. Neumann, Single-Mode Fibers (Springer, 1988).

30. K. Schätzel, "Noise on photon correlation data: I. Autocorrelation functions," Quantum Opt. 2, 287-305 (1990).

31. I. Flammer and J. Rička, "Dynamic light scattering with single-mode receivers: partial heterodyning regime," Appl. Opt. 36, 7508-7517 (1997).

32. D. J. Pine, D. A. Weitz, J. X. Zhu, and E. Herbolzheimer, "Diffusing-wave spectroscopy: dynamic light scattering in the multiple scattering limit," J. Phys. (Paris) 18, 2101-2127 (1990).

33. D. E. Koppel, "Statistical accuracy in fluorescence correlation spectroscopy," Phys. Rev. A 10, 1938-1945 (1974).

34. K. Schätzel, "Noise in photon correlation and photon structure functions," Opt. Acta 30, 155-166 (1983).

35. S. Wray, M. Cope, D. T. Delpy, J. S. Wyatt, and E. O. R. Reynolds, "Characteristics of the near infrared absorption spectra of cytochrome $a a_{3}$ and hemoglobin for the noninvasive monitoring of cerebral oxygenation," Biochim. Biophys. Acta 933, 184-192 (1988).

36. E. Okada and D. T. Delpy, "Near-infrared light propagation in an adult head model. II. Effect of superficial tissue thickness on the sensitivity of the near-infrared spectroscopy signal," Appl. Opt. 42, 2915-2922 (2003).

37. S. Feng, F. Zeng, and B. Chance, "Photon migration in the presence of a single defect: a perturbation analysis," Appl. Opt. 34, 3826-3837 (1995).

38. R. D. Bauer and R. Busse, "Biophysik des Kreislaufs," in Kreislaufphysiologie, R. Busse, ed. (Thieme, 1982), pp. 3-40. 